\title{
Economia, Tecnologia e Saúde
}

\section{Cid Manso de Mello Vianna I}

As tecnologias médicas, nas últimas décadas, têm contribuído significativamente para o aumento da longevidade e da melhor qualidade de vida, como também para o crescimento do custo dos cuidados de saúde. Duas perguntas se tornaram centrais na busca de uma maior eficiência na utilização dos recursos disponíveis para a saúde: "qual é o nível ótimo de gastos em saúde?" e "como a sociedade poderia definir o que é melhor?".

Avaliações econômicas tornaram-se parte integrante do processo de decisões políticas sobre a utilização das tecnologias em saúde. Os principais instrumentos de análise utilizados, cujos exemplos pode ser encontrados neste número, se baseiam na avaliação do custo de oportunidade de estratégias terapêuticas distintas, no custo da utilização dos recursos de uma doença e no impacto orçamentário da incorporação de tecnolgias pelo sistema de saúde.

Abrindo o tema, Pontarolo et al. realizam uma avaliação econômica das anticitocinas adalimumabe (ADA), etanercepte (ETA) e infliximabe (IFX) para o tratamento da artrite reumatóide no Estado do Paraná, sob a perspectiva do Sistema Único de Saúde (SUS). Mosegui et al. avaliam o custo-efetividade e o impacto orçamentário de tratamentos indicados para adultos infectados com genótipo 1 do vírus da hepatite $\mathrm{C}$, comparando o não-tratamento com terapias combinadas de alfapeguinterferon-2a e $2 b$ associados a ribavirina. Fernandes et al. estimam o custo direto médico do tratamento hospitalar de pacientes idosos com fraturas de fêmur proximal, no Hospital Municipal Lourenço Jorge, na cidade do Rio de Janeiro. Cardoso et al. analisam os aspectos clínicos e socioeconômicos decorrentes da presença de dislipidemias portadores de doenças cardiovasculares (DCV). Silva et al. estimam o impacto orçamentário da ampliação da oferta do tratamento da infecção pelo vírus da hepatite $\mathrm{C}$ (VHC) para candidatos a transplante renal.

Nos temas livres, Freitas et al. analisam programas de promoção da saúde e prevenção de doenças em uma operadora de autogestão, com o intuito de compreender se eles disparam dispositivos biopolíticos. Ferreira descreve o impacto do progresso das neurociências, em particular da descoberta dos neurônios-espelhos 
sobre as teses referentes à moralidade. Cavaliere e Costa examinam experiências de isolamento social vividas por indivíduos portadores de hanseníase. Souza e Menandro apresentam uma revisão de bibliografia internacional sobre a Atenção Primária à Saúde, especialmente o cotidiano de trabalho de seus profissionais, suas diretrizes e seus desafios políticos e clínicos. Arce et al. avaliam as práticas de Saúde Mental na atuação das equipes da Estratégia Saúde da Família (ESF) de Brazlândia, no Distrito Federal, quanto a seus potenciais e limites para o cuidado integral aos sujeitos em situações de sofrimento mental na atenção primária. Salles e Barros buscam conhecer a vida cotidiana de egressos de hospitais psiquiátricos, que foram reinternados em um período inferior a seis meses. Silva et al. analisam a satisfação dos pacientes em tratamento hemodialítico crônico com o cuidado recebido nos serviços de diálise. Chazan aborda as práticas e ideias envolvidas na formação acadêmica de um grupo social relevante: o dos médicos. Peduzzi et al. verificam como a contribuição da ação gerencial nos serviços de saúde pode promover uma prática interprofissional. Lima et al investigam os significados atribuídos por pacientes portadores do vírus da hepatite $\mathrm{C}$ sobre a doença e o tratamento dietético. Marques et al estudam o conhecimento e a aceitação das terapias integrativas e complementares e atenção farmacêutica por parte dos usuários do SUS. Junqueira et al. descrevem o cumprimento, em oito municípios do Estado de São Paulo, dos compromissos concernentes à gestão do trabalho inscritos no Pacto de Gestão, assinado em 2006 por gestores dos três níveis do SUS. Geldstein et al. apresentam os resultados parciais de um estudo multicêntrico sobre aspectos socioculturais e demográficos dos acidentes de trânsito na Argentina entre 2004 e 2008. Lima e Büchele fazem uma revisão crítica da literatura nacional e internacional sobre intervençôes com homens autores de violência (HAV) doméstica e familiar contra a mulher. Fechando este número, Elia e Nascimento produzem um estudo de caso sobre o processo de planejamento local em três equipes de Saúde da Família situadas em diferentes áreas programáticas do município do Rio de Janeiro. 